\title{
Prévention des pneumonies acquises sous ventilation mécanique invasive : la sonde, solution à tous les problèmes?
}

\author{
Ventilator-Acquired Pneumonia Prevention: is Endotracheal Tube the Ultimate Solution?
}

\section{F. Philippart}

Reçu le 31 juillet 2015 ; accepté le 27 octobre 2015

(C) SRLF et Lavoisier SAS 2015

Malgré les progrès indéniables de la réanimation au cours des dernières décennies, les pneumonies acquises sous ventilation mécanique (PAVM) forment une entité dont l'éradication, pour opiniâtres que soient les tentatives, reste tenue en échec, avec une prévalence de l'ordre de $15 \%$ des patients ventilés [1]. L'élément central de cette affection restant le passage des pathogènes depuis la sphère ORL vers les voies aériennes inférieures [2], la sonde d'intubation a été l'objet de toutes les attentions.

L'imprégnation de la sonde elle-même par une substance antiseptique, notamment le nitrate d'argent, permet une efficacité partielle, mais les travaux dans ce domaine restent peu nombreux, l'efficacité transitoire et les populations étudiées, ventilées sur de courtes durées, laissant une large place à de nouvelles recherches. L'évacuation des sécrétions s'accumulant au-dessus du ballonnet de la sonde d'intubation a également fait l'objet de nombreux travaux. La réduction par aspiration de la quantité de sécrétions a permis une réduction de l'incidence des PAVM [3,4]. Récemment, l'étude randomisée de Damas et al. incluant plus de 350 patients a retrouvé une réduction de l'incidence des PAVM de l'ordre de neuf patients tous les 100 patients ventilés, soit d'environ $50 \%$ $(8,8 \%$ vs $17,6 \% ; p=0,018)$ [5]. Le caractère continu ou intermittent de cette aspiration reste débattu, mais l'étude menée par Seguin et Laviolle (NCT01555229) pourrait permettre de répondre à cet aspect du problème.

Le ballonnet semble être quant à lui plus une solution qu'un problème dans ce contexte du fait de son étanchéité théorique. Cependant, le polychlorure de vinyle (PVC) favorise la formation de repli qui va permettre l'écoulement des sécrétions par gravité vers les voies aériennes inférieures.

\section{F. Philippart ( $\square)$}

Service de médecine intensive et réanimation, groupe hospitalier Paris Saint Joseph, Paris, France e-mail : fphilippart@gmail.com
Le développement de sondes munies d'un ballonnet en polyuréthane, beaucoup plus fin que le PVC et permettant de ce fait une quasi-disparition de l'écoulement de liquide sur les modèles en bancs a été porteur de nombreux espoirs. Les études cliniques initiales étaient encourageantes, le polyuréthane semblant réduire l'incidence des PAVM, notamment en chirurgie cardiaque. De manière similaire, la modification de la forme du ballonnet, passant d'un cylindre à une forme tronconique permettait de réduire le passage d'un produit de contraste en période peropératoire, confirmant ainsi une meilleure étanchéité du ballonnet. L'association d'une modification de la forme et du matériau est cependant décevante. Dans un travail récent comparant l'efficacité des sondes munies de ballonnet cylindrique ou tronconique, en PVC ou en polyuréthane, aucune différence de colonisation trachéale n'a pu être mise en évidence [6]. L'incidence des PAVM n'était pas davantage modifiée par un type de ballonnet (log-rank test, $\mathrm{P}=0,28$ ) [6]. Ces observations confirment une étude précédente, monocentrique, comparant le ballonnet cylindrique en PVC aux ballonnets cylindrique ou tronconique en polyuréthane [7].

L'efficacité potentielle de ces méthodes dépend néanmoins du maintien de la pression dans le ballonnet luimême. Une synthèse récente des travaux existants par Rouzé et al. mettait en évidence un bénéfice probable d'un contrôle permanent de la pression, même si la réalisation de travaux randomisés, multicentriques était préconisée pour confirmer les données actuellement disponibles [8].

Si de nombreuses hypothèses sont particulièrement intéressantes, il convient probablement de garder en mémoire que c'est l'association de différentes modifications de la sonde d'intubation qui permettra la plus grande réduction du risque de PAVM, jusqu'à voir leur incidence se réduire enfin de manière significative. Ainsi, l'étude américaine, dirigée par Treggiani, permettra peut-être de mettre en évidence l'intérêt de l'association d'un système d'aspiration sousglottique et d'un ballonnet en polyuréthane, même si le dessin de l'étude ne permettra pas d'affirmer la supériorité du 
polyuréthane sur le PVC (NCT01744483). Plus largement, c'est bien évidemment l'usage simultané des différentes méthodes de prévention (mesures générales d'hygiène, aspirations trachéales régulières, soins de bouche réguliers avec ou sans brossage des dents, maintien d'un niveau de pression positive suffisant, position demi-assise du patient, limitation des traitements par inhibiteurs de la pompe à proton pour réduire la colonisation digestive), qui offre la réduction du risque de survenue d'une PAVM la plus importante.

Liens d'intérêts : L'auteur déclare ne pas avoir de lien d'intérêt.

\section{Références}

1. Bekaert M, Timsit JF, Vansteelandt S, et al (2011) Attributable mortality of ventilator-associated pneumonia: a reappraisal using causal analysis. Am J Respir Crit Care Med 184:1133-9
2. Mietto C, Pinciroli R, Patel N, Berra L (2013) Ventilator associated pneumonia: evolving definitions and preventive strategies. Respir Care 58:990-1007

3. Muscedere J, Rewa O, McKechnie K, et al (2011) Subglottic secretion drainage for the prevention of ventilator-associated pneumonia: a systematic review and meta-analysis. Crit Care Med 39:1985-91

4. Frost SA, Azeem A, Alexandrou E, et al (2013) Subglottic secretion drainage for preventing ventilator associated pneumonia: a metaanalysis. Aust Crit Care 26:180-8

5. Damas P, Frippiat F, Ancion A, et al (2015) Prevention of ventilator-associated pneumonia and ventilator-associated conditions: a randomized controlled trial with subglottic secretion suctioning. Crit Care Med 43:22-30

6. Philippart F, Gaudry S, Quinquis L, et al (2015) Randomized intubation with polyurethane or conical cuffs to prevent pneumonia in ventilated patients. Am J Respir Crit Care Med 191:637-45

7. Mahmoodpoor A, Peyrovi-far A, Hamishehkar H, et al (2013) Comparison of prophylactic effects of polyurethane cylindrical or tapered cuff and polyvinyl chloride cuff endotracheal tubes on ventilator-associated pneumonia. Acta Med Iran 51:461-6

8. Rouze A, Jaillette E, Nseir S (2014) Continuous control of tracheal cuff pressure: an effective measure to prevent ventilator-associated pneumonia? Crit Care 18:512 